PROCEEDINGS OF THE

AMERICAN MATHEMATICAL SOCIETY

Volume 102, Number 1, January 1988

\title{
RELATION BETWEEN RIGHT AND LEFT INVOLUTIONS OF A HILBERT ALGEBRA
}

\author{
P. P. SAWOROTNOW
}

(Communicated by John B. Conway)

\begin{abstract}
Existence of a densely defined right involution in a Hilbert algebra implies existence of a left involution.
\end{abstract}

$H^{*}$-algebras were introduced by W. Ambrose [1] to characterize Hilbert-Schmidt operators. This notion was generalized by M. F. Smiley [7], who showed that the structure theorems are valid also for a right $H^{*}$-algebra (a Hilbert algebra whose involution $x \rightarrow x^{r}$ satisfies the condition " $(y x, z)=\left(y, z x^{r}\right)$ " but not the condition $\left."(x y, z)=\left(y, x^{r} z\right) "\right)$. (Hilbert algebra here is a Banach algebra with a Hilbert space norm.) The author showed in [5, Theorem 2] that a proper right $H^{*}$-algebra is also a left $H^{*}$-algebra, i.e. it also has another involution $x \rightarrow x^{l}$ (a left involution) which satisfied the condition " $(x y, z)=\left(y, x^{l} z\right)$."

In this paper we shall show that the same is true also for the case when the involution $x \rightarrow x^{r}$ (the right involution) is defined on a dense subset only.

DEFINITION. Let $A$ be a Hilbert algebra ( $A$ is a Banach algebra with a scalar product (, ) such that $\left.(x, x)=\|x\|^{2}\right)$. We shall say that $A$ is a weak right $H^{*}$ algebra if there is a dense subset $D_{r}$ of $A$ with the property that for each $x \in D_{r}$ there is some $x^{r}$ such that $(y x, z)=\left(y, z x^{r}\right)$ for all $y, z \in A$. We define weak left $H^{*}$-algebra in a similar fashion.

The algebra $A$ is said to be proper if each $x^{r}$ is unique, i.e. $A$ has a right involution $x \rightarrow x^{r}$, defined on a dense subset. Note that $A$ is proper if $r(A)=\{u \in A: A u=$ (0) $\}$ consists of zero alone. Algebra $A$ in Example 2, p. 54, of [5] is an example of weak right (as well as left) $H^{*}$-algebra. Also it is easy to show that each weak right $H^{*}$-algebra is a right complemented algebra.

THEOREM. Each proper weak right $H^{*}$-algebra $A$ is a proper weak left $H^{*}$ algebra.

ProOF. Note that the involution $x \rightarrow x^{r}$ of $A$, defined on a dense subset $D_{r}$, is closable, i.e. the closure of its graph is a graph of some mapping: it is easy to show that if $x_{n} \in D^{r}, x_{n} \rightarrow 0$ and $x_{n}^{r} \rightarrow y \in A$, then $y=0$ (see end of section 8 in $\S 5$, Chapter I of [3]).

Now replace the scalar product (, ) of $A$ and the multiplication $\lambda x$ of members $x$ of $A$ and complex number $\lambda$ by $[$,$] and \lambda \circ x$ respectively, where these new

Received by the editors November 3, 1986.

1980 Mathematics Subject Classification (1985 Revision). Primary 46K15; Secondary 46H20, $47 \mathrm{C} 10$.

Key words and phrases. Hilbert algebra, $H^{*}$-algebra, right $H^{*}$-algebra, left $H^{*}$ - algebra, weak right $H^{*}$-algebra, weak left $H^{*}$-algebra, right involution, left involution. 
products are defined as follows:

$$
\begin{array}{ll}
{[x, y]=(y, x),} & x, y \in A, \\
\lambda \circ x=\bar{\lambda} x, & \lambda \text { is a complex number. }
\end{array}
$$

Let $A^{\prime}$ denote the algebra consisting of members of $A$ but with these new operations. Note that $A^{\prime}$ is also a weak right $H^{*}$-algebra.

Let $T^{\prime}: A \rightarrow A$ be a mapping defined by $T^{\prime} x=x^{r}$ and let $T$ be the closed extension of $T^{\prime}$ (we remarked above that the map $x \rightarrow x^{r}$ is closable). It follows from II in section 9 of $\S 5$ in Chapter I of [3], that $T$ has an adjoint $T^{*}$ defined on a dense subset $D_{l}$ of $A^{\prime}$. We define $x^{l}=T^{*} x$ for each $x \in D_{l}$. It follows that $\left(x^{l}, y\right)=\left(T^{*} x, y\right)=[x, T y]=\left(y^{r}, x\right)$ for all $y \in D_{r}$, from which we see that $\left(x^{l} y, z\right)=\left(x^{l}, z y^{r}\right)=\left(\left(z y^{r}\right)^{r}, x\right)=\left(y z^{r}, x\right)=(y, x z)$ for all $y, z \in D_{r}$ (and each $\left.x \in D_{l}\right)$.

We leave it to the reader to deduce that $(x y, z)=\left(y, x^{l} z\right)$ for all $y, z \in A$.

To conclude the paper it is appropriate to comment that, in the presence of semisimplicity of the algebra $A$, the above Theorem follows from Theorem 2 of [6]. However, semisimplicity of a proper weak right $H^{*}$-algebra would be much more difficult to establish (if it is true at all) than in the case of a right $H^{*}$-algebra. In the latter case it follows from the fact that each ideal (whether closed or not) contains a (right) selfadjoint element (it is easy to show that a selfadjoint element is not a generalized nilpotent).

\section{REFERENCES}

1. W. Ambrose, Structure theorems for a special class of Banach algebras, Trans. Amer. Math. Soc. 57 (1945), 365-386.

2. L. H. Loomis, An introduction to abstract harmonic analysis, Van Nostrand, 1953.

3. M. A. Naimark, Normed rings, "Nauka", Moscow, 1968; English transl., Normed algebras, Wolters-Noordhoff, Groningen, 1972.

4. Frigyes Riesz and Bela Sz.-Nagy, Functional analysis, Ungar, New York, 1955.

5. P. P. Saworotnow, On a generalization of the notion of $H^{*}$-algebra, Proc. Amer. Math. Soc. 8 (1957), 49-55.

6. Math. Soc. 8 (1957), 56-62.

7. M. F. Smiley, Right $H^{*}$-algebra, Proc. Amer. Math. Soc. 4 (1953), 1-4.

Department of Mathematics, The Catholic University of America, WashINGTON, D.C. 20064 